\title{
On Blowup for Gain-Term-Only classical and relativistic Boltzmann equations
}

\author{
HÅKAN ANDRÉASSON* \\ Simone CALOGERo \\ REINHARD ILLNER
}

\begin{abstract}
We show that deletion of the loss part of the collision term in all physically relevant versions of the Boltzmann equation, including the relativistic case, will in general lead to blowup in finite time of a solution and hence prevent global existence. Our result corrects an error in the proof given in Ref. [12, where the result was announced for the classical hard sphere case; here we give a simpler proof which applies much more generally.
\end{abstract}

Key words. Boltzmann equation, relativistic Boltzmann equation, blowup

AMS subject classifications. 76P05, 83A05, 35S30

\section{Introduction}

It has been known for some time that the classical Boltzmann equation admits global unique solutions in all space if either the initial data are small enough in suitable norms, or, equivalently, the mean free path is large enough. The first result of this type appeared in [11, where the analysis was restricted to the hard sphere case. Hamdache [10] generalized the method to cut-off hard potential interactions, and Bellomo and Toscani found a variation of the method which allowed inclusion of less rapidly decreasing initial values [3. The method and the key references may be found in research monographs such as [6] or in the survey article by Ukai [16]. The regularity of these solutions has more recently been explored in work by Mischler and Perthame 14, Boudin and Desvillettes [2].

\footnotetext{
*Department of Mathematics, Chalmers University of Technology, S-412 96 Göteborg, Sweden

$\dagger$ Max-Planck-Institut für Gravitationsphysik, Am Mühlenberg 1, D-14476 Golm, Germany

${ }^{\ddagger}$ Department of Mathematics and Statistics, University of Victoria, Victoria, B.C. V8W 3P4, Canada
} 
The key property which made these global existence and uniqueness results possible is the hidden convolution structure present in the gain part of the collision term; this structure was first observed, and exploited in the discrete velocity setting in one dimension by Tartar [15].

Casual analysis of the quoted literature shows that the method does actually barely use the decomposition of the Boltzmann collision operator into a gain and a loss part; in fact, the results remain true for gain-term-only Boltzmann equations, i.e., equations where the loss part is simply deleted from the collision term. However, no such global result has been proved for the relativistic Boltzmann equation. Here the collision geometry is more involved (see the end of this section) and the available methods of proof in the classical case depend crucially on the collision geometry, eg. the method of Illner and Shinbrot relies on the identity (3.5) in [11, which is a consequence of the Galilean invariance of the classical collision geometry. (See also the discussion in Section 2.5 of [7]).

We remark that it has been known for a long time that in each case where the gain-term-only Boltzmann equation can be solved uniquely on some time interval, then the full Boltzmann equation can be solved, uniquely, on the same time interval for the same initial value. A good method to prove this is the Kaniel-Shinbrot iteration scheme (see [13). The methods in [11 and the other mentioned references really first focus on gain-term only equations. Once these are solved, it is then a rather mechanical matter to include the loss terms.

The methods will certainly not work for spatially homogeneous gain-termonly Boltzmann equations, because (unless one considers zero data) the spatial homogeneity implies that the total amount of gas present is infinite. One of the results we present below is a simple argument which shows that we will in general have blowup for the homogeneous case in finite time even if velocity space is artificially truncated to a bounded domain.

Here, we demonstrate the limits of the method by showing that deletion of the loss term will in general mean loss of global solvability for all relevant space-dependent versions of the Boltzmann equation, including the relativistic case. Such a result was proved in [12] for classical hard spheres, but, as recently observed by one of the authors (H. A.), the proof given there is flawed. Specifically, the method in [12] uses integration of suitably chosen subsets of velocity space to obtain lower bounds for solutions. Near the bottom of page 256 of the reference, a set $\mathcal{M}_{>}^{+}$is defined to complete a certain estimate. A careful geometric analysis shows that this set is always empty, and the argument collapses at this point. R. Illner, co-author of the reference, regrets this error, but is pleased to present a better method in this paper.

The key steps presented in Section 2 below depend mostly on the collision geometry, which is quite simple in the classical cases: Post- (or pre-) collisional velocities lie opposite on a sphere with center $1 / 2(v+w)$ and diameter $\|v-w\|$, where $v$ and $w$ are the velocities of the collision pair under consideration. For relativistic interactions the collision geometry is different from the classical one, due to the fact that the collison invariants have a different form. The collision geometry for classical interactions is spherical and invariant under translations 
(Galilean invariant), i.e., the collision sphere is unchanged as long as the relative velocity remains the same. This is not the case in the relativistic situation where the collision geometry is Lorentz invariant; instead of spheres we get ellipsoids and the excentricity of an ellipsoid changes with the energies of the particles involved in the collision process. The collision geometry is central in Lemma 1 below but the properties of the collision geometry needed in the proof are easily verified in both cases.

\section{The Gain-Term-Only Boltzmann Equation}

We are concerned with the equation

$$
\partial_{t} f+v \cdot \nabla_{x} f=Q_{+}(f, f)
$$

with initial condition

$$
f(0, x, v)=\varphi(x, v) .
$$

Here, $t \geq 0$ denotes time, $\varphi$ is an integrable nonnegative initial value, and

$$
Q_{+}(f, f)(v)=\int_{\Re^{3}} \int_{S_{+}^{2}} B(|v-w|, n \cdot(v-w)) f\left(v^{\prime}\right) f\left(w^{\prime}\right) \mathrm{d} n \mathrm{~d} w
$$

denotes the gain part of the usual collision term for the Boltzmann equation, in standard notation (the local dependence on $t$ and $x$ in (2.3) is omitted). Pre- and post- collisional velocities satisfy the conservation of momentum, $v+w=v^{\prime}+w^{\prime}$, and of energy, $|v|^{2}+|w|^{2}=\left|v^{\prime}\right|^{2}+\left|w^{\prime}\right|^{2}$. $B$ is the collision kernel which depends on the type of interparticle potential. For details, see [4]. In the hard sphere case, considered in $[12, B=n \cdot(v-w)$. For this case it is shown there that the initial value problem has a local unique solution; the point is that this solution will in general blow up in finite time.

Our present objective is to provide a correct and more general proof for this fact. The proof will work for all kernels $B$ satisfying the condition that if $M \in S_{+}^{2} \times \Re^{3}$ is a set of positive measure, then

$$
\iint_{M} B \mathrm{~d} n \mathrm{~d} w>0
$$

This assumption is used in the proof of Lemma 1 below and does not seem to exclude anything physically reasonable. We will further work under the assumption that the initial value problem is uniquely solvable locally in time, a fact which can be proved for reasonable collision kernels along the lines of the proof in [13].

As in [12] we restrict our analysis to initial values $\varphi(x, v)=c_{0} \chi_{1}(x) \chi_{2}(v)$ where $\chi_{1}$ and $\chi_{2}$ are characteristic functions of the balls $B_{1}=\left\{x ;|x| \leq c_{1}\right\}, B_{2}=$ $\left\{v ;|v| \leq c_{2}\right\}$, and $c_{1}, c_{2}$ are positive constants to be chosen later. 
Before we can deal with the full problem, we focus on the spatially homogeneous case with a truncation in velocity space. The reason for the truncation will become transparent later.

A key ingredient in our argument is the following estimate for the action of the gain term on characteristics functions. ${ }^{1}$

Lemma 1 Let $B_{R}=\{v ;|v| \leq R\}$ and assume that $B$ satisfies 2.4). Then there are constants $\lambda>1$ and $\delta>0$ such that

$$
Q_{+}\left(\rho \chi_{B_{R}}, \rho \chi_{B_{R}}\right)(v) \geq \delta \rho^{2} \chi_{\lambda B_{R}}(v) \geq \delta \rho^{2} \chi_{B_{R}}(v) .
$$

Proof: This estimate is a consequence of the collision geometry, see Figure 1 below (one dimension is suppressed). ${ }^{2}$ Choosing $v$ in or just outside the ball $B_{R}$, we can construct collision spheres such that both the centre of the sphere and the antipode $w$ of $v$ are both in $B_{R}$, and the post-collisional velocities $v^{\prime}, w^{\prime}$ are also in $B_{R}$ for a set of collision parameters $n \in S_{+}^{2}$ of positive measure on $S^{2}$. The constant $\delta$ is a positive lower bound of $I_{v}:=c \iint_{M} B(|v-w|, n \cdot(v-w)) \mathrm{d} n \mathrm{~d} w$, where $M$ is the positive measure set of $w \in B_{R}$ and $n \in S_{+}^{2}$ for which the above conditions hold. It is clear that $I_{v}$ has a positive lower bound unless $\lambda$ is chosen too large or unless $B$ vanishes on sets of positive measure, which is prohibited by the assumption (2.4).

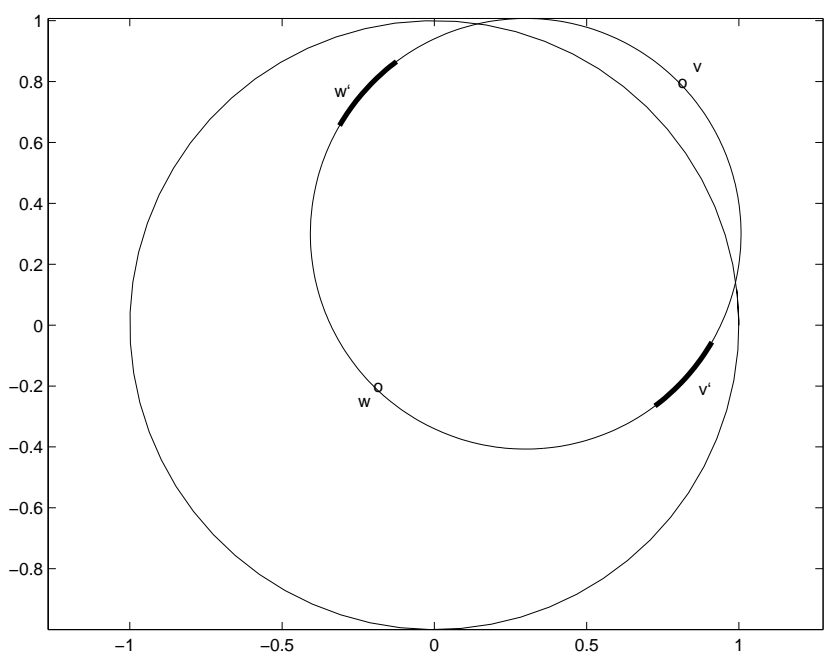

Figure 1: Non-relativistic collision geometry

The proof of the next lemma is immediate, but we formulate the result for easy reference.

\footnotetext{
${ }^{1}$ We are grateful to Cedric Villani for pointing out this estimate to us.

${ }^{2}$ Figures have been obtained using the software Matlab.
} 
Lemma 2 Let $f \geq g \geq 0$ be integrable density functions for which $Q_{+}$is defined. Then $Q_{+}(f, f)(v) \geq Q_{+}(g, g)(v)$.

Next, we define $Q_{R}(f, f)(v)=\inf _{v}\left\{\chi_{\{|v| \leq R\}} Q_{+}(f, f)(v)\right\}$. Note that $Q_{R}$ is defined such that $Q_{R}(v)=0$ if $|v|>R$, and that $Q_{R}(v)$ takes a constant value for $|v| \leq R$.

Consider an initial value $f_{0}=f_{0}(|v|)$ (the isotropy in $v$ is not essential but convenient) such that there is a constant $\rho_{0}>0$ with $f_{0}(|v|) \geq \rho_{0} \chi_{B_{R}}(v)$. Let $f(t, v)$ be the (local) nonnegative solution of

$$
\frac{d}{d t} f=Q_{+}(f, f), \quad f(0, v)=f_{0}(v)
$$

and let $\tilde{f}(t, v)$ be the solutions of

$$
\frac{d}{d t} \tilde{f}=Q_{R}(\tilde{f}, \tilde{f}), \quad \tilde{f}(0, v)=\rho_{0} \chi_{B_{R}}(v) .
$$

\section{Lemma 3}

$$
f(t, v) \geq \tilde{f}(t, v)
$$

while $f(t, v)$ exists.

Proof: Let $[g-h]_{+}$equal $g-h$ if $g>h$ and 0 otherwise. We derive a Gronvall inequality for $\left\|[\tilde{f}(t, \cdot)-f(t, \cdot)]_{+}\right\|_{\infty}$ which shows that it remains zero as long as the solution exists.

We have

$$
\begin{aligned}
\left\|[\tilde{f}(t, \cdot)-f(t, \cdot)]_{+}\right\|_{\infty} & =\left\|\left[\tilde{f}_{0}(v)-f_{0}(v)+\int_{0}^{t} Q_{R}(\tilde{f}, \tilde{f})-Q_{+}(f, f) \mathrm{d} s\right]_{+}\right\|_{\infty} \\
& \leq \int_{0}^{t}\left\|\left[Q_{+}(\tilde{f}, \tilde{f})-Q_{+}(f, f)\right]_{+}\right\|_{\infty} \mathrm{d} s
\end{aligned}
$$

by the definition of $Q_{R}$. The integrand in (2.8) can be written and estimated as follows

$$
\begin{gathered}
\left\|\left[\iint B \tilde{f}\left(v^{\prime}\right)\left(\tilde{f}\left(w^{\prime}\right)-f\left(w^{\prime}\right)\right)+B f\left(w^{\prime}\right)\left(\tilde{f}\left(v^{\prime}\right)-f\left(v^{\prime}\right)\right) \mathrm{d} n \mathrm{~d} w\right]_{+}\right\|_{\infty} \\
\leq C\left\|[\tilde{f}(t, \cdot)-f(t, \cdot)]_{+}\right\|_{\infty},
\end{gathered}
$$

where

$$
C=\left\|\iint B \tilde{f}\left(v^{\prime}\right) \mathrm{d} n \mathrm{~d} w\right\|_{\infty}+\left\|\iint B f\left(w^{\prime}\right) \mathrm{d} n \mathrm{~d} w\right\|_{\infty} .
$$

Since $C$ is finite on the interval of existence the claim follows from (2.8).

Lemma 4 The solution of 2.7) is of the form $\tilde{\rho}(t) \chi_{B_{R}}(v)$.

Proof: This follows because $\tilde{f}(0, v)=\rho_{0} \chi_{B_{R}}(v)$ and because $Q_{R}(\tilde{f}, \tilde{f})$ is homogeneous on $B_{R}$ by construction, and zero elsewhere. 
Theorem $1 \tilde{f}$, and hence $f$, both blow up in finite time.

Proof: For any $v \in B_{R}$,

$$
\begin{aligned}
\frac{d}{d t} \tilde{f}(v) & =\frac{d}{d t} \tilde{\rho}(t) \\
& =Q_{R}(\tilde{f}, \tilde{f}) \\
& \geq \inf _{|v| \leq R} Q_{+}\left(\tilde{\rho} \chi_{B_{R}}, \tilde{\rho} \chi_{B_{R}}\right) \\
& \geq \delta \tilde{\rho}^{2}(t) .
\end{aligned}
$$

Hence $\frac{d}{d t} \tilde{\rho} \geq \delta \tilde{\rho}^{2}$, and the assertion follows.

We return now to the spatially inhomogeneous case. The rest of our argument is identical to what was done on [12, but we repeat the analysis here for the convenience of the reader. First, recall that we are concerned with

$$
\partial_{t} f+v \cdot \nabla_{x} f=Q_{+}(f, f),
$$

with initial values $\varphi(x, v)=c_{0} \chi_{1}(x) \chi_{2}(v)$. It is known 13 that the initial value problem has a unique nonnegative mild local solution under reasonable assumptions on the collision kernel $B$. Moreover, if we consider the modified problem

$$
\partial_{t} \hat{f}+v \cdot \nabla_{x} \hat{f}=\chi_{2}(v) Q_{+}(\hat{f}, \hat{f}), \hat{f}(0, x, v)=\varphi(x, v),
$$

then this problem is also uniquely solvable in the mild sense, and $f \geq \hat{f}$ while the larger of the two exists. To show blowup it is therefore enough to show that $\hat{f}$ will blow up in finite time, and to this end we will reduce the problem to the spatially homogeneous case resolved in the previous section. We need

Lemma 5 Choose $T>0$ arbitrary but fixed, and suppose that $c_{1}>T c_{2}$. Then, if $0 \leq t<T$ is in the interval of existence of $\hat{f}$, and if $|x|,|y| \leq c_{1}-t c_{2}$, then $\hat{f}(t, x, v)=\hat{f}(t, y, v)$ for all $v$.

Proof: This proof is identical to the one in 12. First, note that the local solution of the initial value problem is obtained as the limit as $k \rightarrow \infty$ of the iteratively defined functions $\hat{f}_{k}$, where $\hat{f}_{0}=0$ and

$$
\begin{aligned}
& \hat{f}_{k+1}(t, x, v)=c_{0} \chi_{1}(x-t v) \chi_{2}(v) \\
& +\chi_{2}(v) \int_{0}^{t} \iint B \hat{f}_{k}\left(\tau, x-(t-\tau) v, v^{\prime}\right) \hat{f}_{k}\left(\tau, x-(t-\tau) v, w^{\prime}\right) \mathrm{d} n \mathrm{~d} w .
\end{aligned}
$$

We also note that $\hat{f}_{k+1} \geq \hat{f}_{k}$, and by construction $f_{k}(v)=0$ for all $k$ and all $|v|>c_{2}$. The claim of the lemma is trivial for $k=0$, and assuming that it holds for some $k>0$, it follows from (2.12) that it also holds for $k+1$ : in fact, if one of $\left|v^{\prime}\right|$ or $\left|w^{\prime}\right|$ is larger than $c_{2}$, the integrand in (2.12) vanishes. If $\max \left\{\left|v^{\prime}\right|,\left|w^{\prime}\right|\right\} \leq$ $c_{2}$, and $|x| \leq c_{1}-t c_{2}$, we have in addition that $|x-(t-\tau) v| \leq|x|+(t-\tau) c_{2} \leq$ $c_{1}-\tau c_{2}$. The inductive hypothesis then entails that

$$
\hat{f}_{k}(t, x-(t-\tau) v, \zeta)=\hat{f}_{k}(t, y-(t-\tau) v, \zeta)
$$


for both $\zeta=v^{\prime}, \zeta=w^{\prime}$ whenever $|x|,|y| \leq c_{1}-t c_{2}$. It follows that for all such $x, y$, all $v$ and all $t \in[0, T]$ that $\hat{f}_{k+1}(t, x, v)=\hat{f}_{k+1}(t, y, v)$.

The next Theorem is our main result.

Theorem 2 Let $f$ be the local mild solution of (2.10) with the initial value $\varphi(x, v)=c_{0} \chi_{1}(x) \chi_{2}(v)$. If $c_{0}$ or $c_{1}$ are sufficiently large this solution will blow up in finite time, in both the $L^{1}-$ or the $L^{\infty}-$ norm.

Proof: If we consider a fixed time interval $[0, T]$ and a fixed $c_{2}>0$, let $c_{1}>T c_{2}$. Then the previous lemma implies that $\hat{f}$ is independent of $x$ in the set $\{(t, x) ; 0 \leq$ $\left.t \leq T,|x| \leq c_{1}-t c_{2}\right\}$, so on this set the evolution of $\hat{f}$ is effectively given as the solution of the initial value problem

$$
\frac{d}{d t} \hat{f}(v)=\chi_{2}(v) Q_{+}(\hat{f}, \hat{f}), \hat{f}(0, v)=c_{0} \chi_{2}(v) .
$$

We know from our earlier discussion of the truncated spatially homogeneous case that the solution of this initial value problem will blow up in $[0, T]$ if $c_{0}$ is large enough; alternatively, if $c_{0}$ is positive but small, we can make $c_{1}$ large enough such that the condition $c_{1}>c_{2} T$ will hold for the required blowup time $T$. The assertion of the theorem follows.

\section{The relativistic case}

This section contains a discussion on the generalization of Theorem 2 to the relativistic Boltzmann equation. We start with a short introduction to the problem, see [5] for more details.

The speed of light and the rest mass of particles are both normalized to unity, i.e., $m=c=1$; the convention on the signature is $(+---)$. We denote by $f=f(t, x, p)$ the one-particle distribution function, with $p \in \Re^{3}$ denoting the momentum variable. The relativistic Boltzmann equation has the form

$$
\left(\partial_{t}+\frac{p}{p_{0}} \cdot \nabla_{x}\right) f=Q(f, f)
$$

where the collision operator is defined by

$$
Q(f, g)(p)=\frac{1}{p_{0}} \int_{\Re^{3}} \int_{S^{2}}\left(f\left(p^{\prime}\right) g\left(q^{\prime}\right)-f(p) g(q)\right) B(g, \theta) \mathrm{d} \Omega \frac{\mathrm{d} q}{q_{0}} .
$$

Here $\mathrm{d} \Omega$ is the element of surface area on $S^{2}, p^{\mu}=\left(p_{0}, p\right)$ is the four momentum $\left(p_{0} \in \Re^{+}, p \in \Re^{3}, \mu=0,1,2,3\right)$, and $p_{0}=\sqrt{1+|p|^{2}}$ is the particle energy. Conservation of momentum and energy now read

$$
p+q=p^{\prime}+q^{\prime}, \quad \sqrt{1+|p|^{2}}+\sqrt{1+|q|^{2}}=\sqrt{1+\left|p^{\prime}\right|^{2}}+\sqrt{1+\left|q^{\prime}\right|^{2}} .
$$


The total energy and the relative momentum in the center of mass system are $s^{1 / 2}=\left|q^{\mu}+p^{\mu}\right|$ and $2 g=\left|q^{\mu}-p^{\mu}\right|$, respectively. The scattering angle $\theta$ in the center of mass system satisfies

$$
\cos \theta=1-2 \frac{\left(p^{\mu}-q^{\mu}\right)\left(p_{\mu}^{\prime}-q_{\mu}^{\prime}\right)}{\left|p^{\mu}-q^{\mu}\right|^{2}}
$$

The kernel $B(g, \theta)$ and the scattering cross section $\sigma(g, \theta)$ are related by

$$
B(g, \theta)=\frac{g s^{1 / 2}}{2} \sigma(g, \theta)
$$

The case of a constant scattering cross section corresponds to the relativistic hard sphere model. For Maxwellian molecules one has $\sigma=g^{-1}\left(1+g^{2}\right)^{1 / 2} F(\theta)$, where $F$ is an arbitrary function of the scattering angle.

We also mention a different representation of the relativistic collision operator which is closer in form to the classical one, see Appendix II in 9 for a derivation. We can write

$$
Q(f, g)=\int_{\Re^{3}} \int_{S^{2}} k(p, q, \omega)\left[f\left(p^{\prime}\right) g\left(q^{\prime}\right)-f(p) g(q)\right] \mathrm{d} \omega \mathrm{d} p
$$

where $p^{\prime}=p+a(p, q, \omega) \omega, q^{\prime}=q-a(p, q, \omega) \omega$ and

$$
\begin{gathered}
k(p, q, \omega)=4 s \sigma\left(p_{0}+q_{0}\right)^{2} \frac{|\omega \cdot(\hat{q}-\hat{p})|}{\left(e^{2}-\left(\omega \cdot(p+q)^{2}\right)\right)^{2}} \\
a(p, q, \omega)=\frac{2 e p_{0} q_{0}(\omega \cdot(\hat{q}-\hat{p}))}{e^{2}-(\omega \cdot(p+q))^{2}} .
\end{gathered}
$$

Here $e:=p_{0}+q_{0}$ is the total energy and $\hat{x}:=x / x_{0}$. As shown in Figure 2 below, for fixed $p, q$ and as $\omega$ varies on $S^{2}, p^{\prime}$ and $q^{\prime}$ lie on an ellipsoid (one dimension is suppressed). The excentricity of the ellipsoid is given by

$$
\alpha=\frac{\sqrt{1+|p|^{2}} \sqrt{1+|q|^{2}}}{\sqrt{1+\left|p^{\prime}\right|^{2}} \sqrt{1+\left|q^{\prime}\right|^{2}}}
$$

In particular it is bounded for bounded pre-collisional momenta.

Finally, the collision operator can in an obvious way be written as

$$
Q(f, g)=Q_{+}(f, g)-Q_{-}(f, g)
$$

where $Q_{+}$and $Q_{-}$are referred to as the gain and loss term respectively. Hence, the gain-term-only relativistic Boltzamnn equation reads,

$$
\left(\partial_{t}+\hat{p} \cdot \nabla_{x}\right) f=Q_{+}(f, f)
$$

Local existence and uniqueness of mild solutions to the Cauchy problem for the relativistic Boltzmann equation has been shown in [1, where the more 
general case of a background curved metric is considered. For initial data close to equilibrium global existence has been obtained in various function spaces [8, 9]. At present a similar result for data near vacuum, i.e., for small data, is not available as was briefly discussed in the introduction.

By inspection of the proof of Theorem 1 it follows that an identical result also holds for the gain-term-only relativistic Boltzmann equation (3.6). The essential properties of the collision geometry needed in the proof of Lemma 1 are easily verified also in this case. The relevant picture in the relativistic case is given by Figure 2 below.

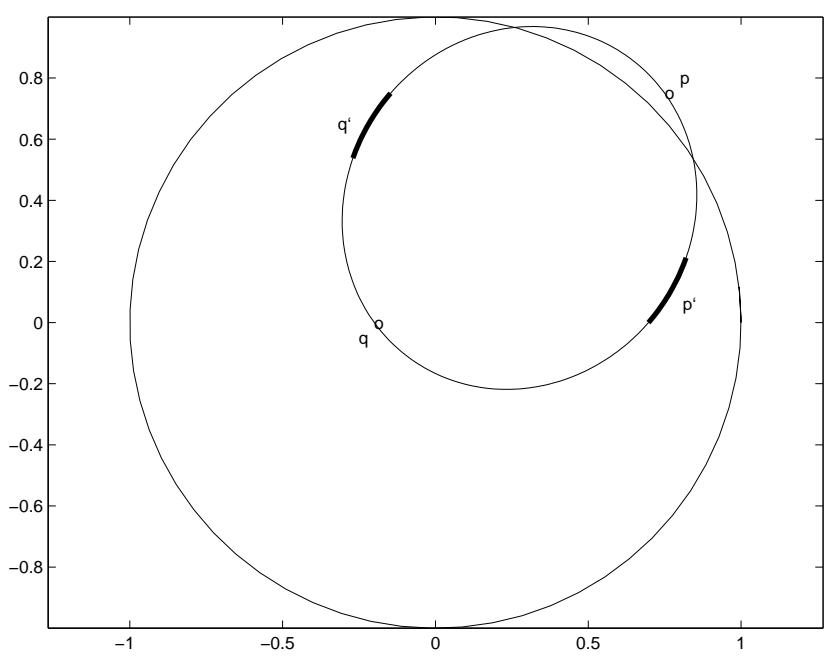

Figure 2: Relativistic collision geometry

The remaining details of the proof of blowup for the only-gain-term relativistic Boltzmann equation are very similar to the classical case and are omitted. Note however, that since finite propagation speed is already present in the relativistic situation, velocity truncation is not longer necessary in this case.

Acknowledgment: S. C. acknowledges support by the European HYKE network (contract HPRN-CT-2002-00282).

\section{References}

[1] K. Bichteler: On the Cauchy Problem of the Relativistic Boltzmann Equation, Comm. Math. Phys. 4, 352-364 (1967)

[2] L. Boudin and L. Desvillettes: On the singularities of the global small solutions of the full Boltzmann equation, Monatsh. Math. 131, 91-108 (2000) 
[3] N. Bellomo and G. Toscani: On the Cauchy problem for the nonlinear Boltzmann equation. Global existence, uniqueness and asymptotic stability, J. Math. Phys. 26, 334-338 (1985)

[4] C. Cercignani: The Boltzmann equation and its application, SpringerVerlag, New York (1988)

[5] C. Cercignani and G. M. Kremer: The Relativistic Boltzmann Equation: Theory and Applications, Birkhäuser, Basel (2002)

[6] C. Cercignani, R. Illner and M. Pulvirenti: The Mathematical Theory of Dilute Gases, Springer-Verlag, New York (1994)

[7] R. T. Glassey: The Cauchy Problem in Kinetic Theory, sIAm, Philadelphia (1994)

[8] R. T. Glassey and W. A. Strauss: Asymptotic Stability of the Relativistic Equilibrium, Publ.R.I.M.S Kyoto Univ. 29, 301-347 (1993)

[9] R. T. Glassey and W. A. Strauss: Asymptotic Stability of the Relativistic Maxwellian via Fourteen Moments, Trans. Th. Stat. Phys. 24, 657-678 (1995)

[10] K. Hamdache: Quelques résultats pour l'équation de Boltzmann, C. R. Acad. Sci. Paris 299, Série I, 431-434 (1984)

[11] R. Illner and M. Shinbrot: The Boltzmann Equation: Global Existence for a Rare Gas in an Infinite Vacuum, Comm. Math. Phys. 95, 217-226 (1984)

[12] R. Illner and M. Shinbrot: Blow-up of solutions of the gain-term only Boltzmann equation, Math. Meth. in the Appl. Sci. 9, 251-259 (1987)

[13] S. Kaniel and M. Shinbrot: The Boltzmann Equation: Uniqueness and local existence, Comm. Math. Phys. 58, 65-84 (1978)

[14] S. Mischler and B. Perthame: Boltzmann equation with infinite energy: renormalized solutions and distributional solutions for small initial data and initial data close to a Maxwellian, SIAM J. Math. Anal. 28, 1015-1027 (1997)

[15] L. Tartar: Some existence theorems for semilinear hyperbolic systems in one space variable, MRC Technical Summary Report, Madison (1980)

[16] S. Ukai: Solutions of the Boltzmann equations, Studies in Math. Appl. 18, 37-96 (1986) 